\title{
CMOS-Compatible PureGaB Ge-on-Si APD Pixel Arrays
}

\author{
Amir Sammak, Mahdi Aminian, Lis K. Nanver, Member, IEEE, and Edoardo Charbon, Senior Member, IEEE
}

\begin{abstract}
Pure gallium and pure boron (PureGaB) Ge-on-Si photodiodes were fabricated in a CMOS compatible process and operated in linear and avalanche mode. Three different pixel geometries with very different area-to-perimeter ratios were investigated in linear arrays of 300 pixels with each a size of $26 \times 26 \mu \mathrm{m}^{2}$. The processing of anode contacts at the anode perimeters leaving oxide covered PureGaB-only light-entrance windows, created perimeter defects that increased the vertical Ge volume but did not deteriorate the diode ideality. The dark current at $1 \mathrm{~V}$ reverse bias was below $35 \mu \mathrm{A} / \mathrm{cm}^{2}$ at room temperature and below the measurement limit of $2.5 \times 10^{-2} \mu \mathrm{A} / \mathrm{cm}^{2}$ at $77 \mathrm{~K}$. Spread in dark current levels and optical gain, that reached the range of $10^{6}$ at $77 \mathrm{~K}$, was lowest for the devices with largest perimeter. All device types were reliably operational in a wide temperature range from $77 \mathrm{~K}$ to room temperature. The spectral sensitivity of the detectors extended from visible to the telecom band with responsivities of 0.15 and $0.135 \mathrm{~A} / \mathrm{W}$ at 850 and $940 \mathrm{~nm}$, respectively.
\end{abstract}

Index Terms-Avalanche photodiode (APD), Ge-on-Si, nearinfrared photodiode, pure gallium and pure boron (PureGaB).

\section{INTRODUCTION}

A VALANCHE photodiodes (APDs) and single-photon avalanche diodes (SPADs), or Geiger-mode APDs, are widely used in optical telecommunications, imaging, and medical diagnostics, where high sensitivity to light in the visible or near-infrared (NIR) ranges is needed [1]. Silicon photomultipliers (SiPMs) and imaging arrays of APDs and SPADs have seen a fast growth in the last decade, and are used today in a variety of applications, including time-offlight imaging, positron emission tomography, fluorescence microscopy, and Raman spectroscopy, to name a few [2], [3]. Nevertheless, photomultiplier tubes, the predecessors

Manuscript received March 15, 2015; revised June 3, 2015; accepted July 11, 2015. Date of publication November 18, 2015; date of current version December 24, 2015. This work was supported in part by the Netherlands Agency, IOP Photonics Devices, through RASKIN Project, and in part by the Swiss Research Program Nano-Tera. The review of this paper was arranged by Editor S. Kawahito. (Amir Sammak and Mahdi Aminian equally contributed to this work.)

A. Sammak is with the Department of Microelectronics, Delft University of Technology, Delft 2628 CT, The Netherlands (e-mail: a.sammak@tudelft.nl).

M. Aminian is with the Department of Electrical Engineering, École Polytechnique Fédérale de Lausanne, Lausanne 1015, Switzerland (e-mail: mahdi.aminian@epfl.ch).

L. K. Nanver is with Aalborg University, Aalborg 9220, Denmark, and also with the University of Twente, Enschede NB 7522, The Netherlands (e-mail: 1.k.nanver@utwente.nl).

E. Charbon is with the Delft University of Technology, Delft 2628 CT, The Netherlands (e-mail: e.charbon@tudelft.nl).

Color versions of one or more of the figures in this paper are available online at http://ieeexplore.ieee.org.

Digital Object Identifier 10.1109/TED.2015.2457241 of SiPMs, are still the devices of choice in many applications due to their good timing resolution and ease of use.

Silicon ( $\mathrm{Si}$ ) photodetectors and CMOS SPADs cannot be used at infrared wavelengths because of the large bandgap that makes them only suitable for wavelengths shorter than $1.1 \mu \mathrm{m}$. These sensors in fact exhibit high sensitivity in the visible range usually peaking at $450-500 \mathrm{~nm}$.

For sensitivity at NIR wavelengths, germanium (Ge) and direct bandgap semiconductors, such as $\mathrm{HgCdTe}$, and the III-V compounds InGaAs/InP are generally used due to their narrower bandgap that gives good absorption properties and performance in this range [4]. These compound materials have a tunable bandgap, allowing detection into the shortwave infrared to the very long wave infrared regions, but none of them have been integrated in CMOS. Ge APDs are cheaper to produce, and have been available for decades, but it has been problematic to fabricate them with the desired sensitivity in the NIR range and sufficiently low noise levels over a wide frequency band. Nevertheless, Ge is attractive because it has been possible to develop processes allowing integration in CMOS, and low-quality Ge photodiodes have been demonstrated in telecom circuits [5], [6]. For highquality devices, the lattice mismatch between $\mathrm{Si}$ and $\mathrm{Ge}$ has meant a fundamental incompatibility with the standard CMOS processing, preventing the implementation of low-cost photon counters and arrays.

The pure gallium and pure boron (PureGaB) Ge-on-Si process, that is the topic of this paper, is an example of a CMOS compatible technology for fabricating high-quality Ge photodiode arrays. In [7]-[10], the fabrication of these photodiodes was described in detail. In short, single-crystalline islands of $\mathrm{Ge}$ are selectively deposited in oxide windows to a Si substrate. The Ge is n-doped in situ with arsenic (As), and to form a $\mathrm{p}^{+}$-region on this $\mathrm{n}-\mathrm{Ge}$, the deposition cycle is continued with the growth of 1-nm-thin layer-stack of PureGaB. Both $\mathrm{Ga}$ and $\mathrm{B}$ are p-type dopants for Ge, and the layer stack of PureGaB has been shown to deliver an ultrashallow junction with low saturation current even though the junction depth is only a few nanometer.

In this paper, the optical characterization of the PureGaB Ge-on-Si photodiodes is investigated for the first time at temperatures from 77 to $300 \mathrm{~K}$. In [7], we describe how a new metallization approach for the fabrication of the PureGaB Ge-on-Si photodiodes was implemented to achieve an oxide covered PureGaB-only light-entrance window on each photodiode in a $300 \times 1$ array in order to obtain the maximum photon absorption. The study was limited to material 


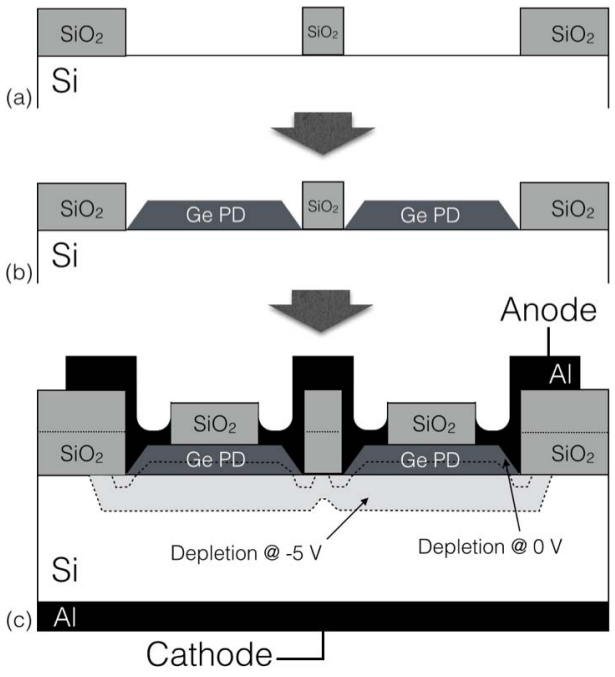

Fig. 1. Schematic of the process flow for fabricating PureGaB Ge-on-Si diodes with the metallization approach presented in [7]. (a) Windows are opened through $\mathrm{SiO}_{2}$ to $\mathrm{Si}$. (b) PureGaB Ge-on-Si photodiodes are deposited in one deposition cycle in the windows. (c) Al-contacts to the anode and cathode regions are made along with $\mathrm{SiO}_{2}$-covered PureGaB-only light-entrance windows.

analysis and electric characterization of the diodes, and revealed that aluminum $(\mathrm{Al})$ contacting of the PureGaB at the diode perimeter could become a tradeoff between preserving the PureGaB barrier to the Al-contact metal and having low series resistance. Nevertheless, it was possible to maintain a good electrical performance with low dark currents and good uniformity across the arrays of $300 \times 1$ devices even when the PureGaB was penetrated by the Al. While the Si-to-Ge interface contained a very low defect level after the PureGaB deposition [8], this contact processing increased defect levels at the diode perimeter. The low dark-current levels that were nevertheless achieved have been ascribed to the damage-free processing of the $\mathrm{p}^{+}$-anode regions. Photodiodes of different sizes and geometries were realized with the good quality $I-V$ characteristics in terms of the ideality factors and dark currents that, to the best of our knowledge, are among the lowest reported in the literature for $\mathrm{Ge}$ and $\mathrm{Ge}-\mathrm{on}-\mathrm{Si}$ diodes [9]. The devices described in this paper were suitable for operation both in linear and avalanche mode. The linear arrays of 300 pixels were designed and fabricated as part of a project targeting low-cost medical diagnostics with Raman spectroscopy in the NIR [11]. Other potential applications include time-of-flight imaging and NIR optical tomography. The optical characterization confirms the electrical result that the arrays have high reproducibility, and the peak sensitivity is at $\sim 850 \mathrm{~nm}$ with a peak optical gain at $940 \mathrm{~nm}$.

\section{Device Design AND Fabrication}

Fig. 1 shows a schematic of the fabrication process of the PureGaB Ge-on-Si arrays. Arrays of $300 \times 1$ pixels were integrated on substrates of 2-5 $\Omega$-cm n-type (100) Si wafers. First, a $30-\mathrm{nm}$ thermal $\mathrm{SiO}_{2}$ is grown on the $\mathrm{Si}$ surface followed by a low-pressure $\mathrm{CVD} \mathrm{SiO}_{2}$ layer deposition with a thickness of $\sim 1 \mu \mathrm{m}$. The $\mathrm{SiO}_{2}$ layer was patterned and

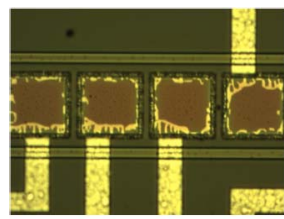

(a)

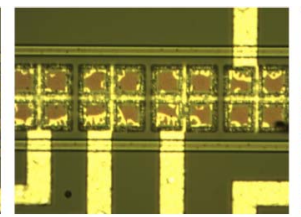

(b)

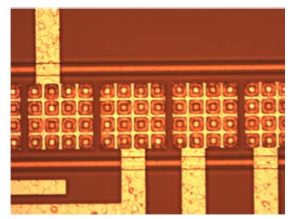

(c)
Fig. 2. Optical images of (a) single-diode pixel of $26 \times 26 \mu \mathrm{m}^{2}$, (b) 4-diode quadpixel composed of $12 \times 12 \mu \mathrm{m}^{2}$ Ge-on-Si photodiodes, and (c) 16-diode hexapixel composed of $5 \times 5 \mu \mathrm{m}^{2} \mathrm{Ge}$-on-Si photodiodes.

TABLE I

Geometrical Parameters of THE THReE DifFERENT PHOTODIODE PIXELS

\begin{tabular}{|l|c|c|c|c|}
\hline & $\begin{array}{c}\text { No. of } \\
\text { diodes / } \\
\text { pixel }\end{array}$ & $\begin{array}{c}\text { Diode } \\
\text { size }[\mu \mathrm{m}]\end{array}$ & $\begin{array}{c}\text { Total diode } \\
\text { area in pixel } \\
{\left[\mu \mathrm{m}^{2}\right]}\end{array}$ & $\begin{array}{c}\text { Total diode } \\
\text { perimeter in } \\
\text { pixel }[\mu \mathrm{m}]\end{array}$ \\
\hline Single pixel & 1 & $26 \times 26$ & 676 & 104 \\
\hline Quad pixel & 4 & $12 \times 12$ & 576 & 192 \\
\hline Hexa pixel & 16 & $5 \times 5$ & 400 & 320 \\
\hline
\end{tabular}

etched on the Si surface by a mask that defined the Ge-on-Si photodiode areas. As illustrated by the microimages of Fig. 2, the arrays were designed with three different pixel geometries: 1) a single-diode pixel of size $\left.26 \times 26 \mu \mathrm{m}^{2} ; 2\right)$ a 4-diode pixel (quad) composed of $12 \times 12 \mu \mathrm{m}^{2}$ Ge diodes; and (3) a 16-diode pixel (hexa) composed of $5 \times 5 \mu \mathrm{m}^{2}$ Ge diodes. All the pixels had the same total area of $26 \times 26 \mu \mathrm{m}^{2}$. However, since the diodes in the multidiode devices were separated by a $1-\mu \mathrm{m}$-wide oxide region, the actual Ge area decreases with the number of diodes while the Ge perimeter increases. The exact geometrical design parameters of the PureGaB photodiodes are listed in Table I. Moreover, around each of the $300 \times 1$ pixel arrays, a set of two 5 - $\mu \mathrm{m}$-wide lines were patterned to prevent $\mathrm{Ge}$ deposited on surrounding oxide regions from reaching the windows designed for the deposition of the As-doped Ge islands. Such undesirable loading effects could otherwise lead to very nonuniform deposition across the array [8]. To also counteract the nucleation of $\mathrm{Ge}$ on the $\mathrm{SiO}_{2}$, the surrounding open $\mathrm{Si}$ area was maximized. These precautions also reduce the loading effects and the growth-rate nonuniformities of the in situ As n-doping [8]. The defect-density of the crystalline Ge that is deposited with this approach is shown to be in the order of $10^{7} \mathrm{~cm}^{-2}$ [12].

The devices were then transferred to a plasma-enhanced CVD (PECVD) reactor for deposition of an 800-nm-SiO 2 layer, which was then patterned with $1-\mu \mathrm{m}$-wide windows along the perimeter of the Ge-on-Si photodiodes. These windows were opened by plasma etching with soft landing on the PureGaB. Next, $800-\mathrm{nm} \mathrm{Al} / \mathrm{Si} 1 \%$ was sputtered and then removed over the photosensitive junctions by means of selective plasma etching of the $\mathrm{Al}$ layer to the oxide covering the PureGaB. As a last step, the metal was alloyed for $1 \mathrm{~h}$ at $400{ }^{\circ} \mathrm{C}$ in forming gas to achieve H-passivation of the $\mathrm{Si} / \mathrm{SiO}_{2}$ interface.

From the images in Fig. 2 of the fabricated pixels, it can be seen that the Al removal on the oxide-covered light-entrance 
(a)

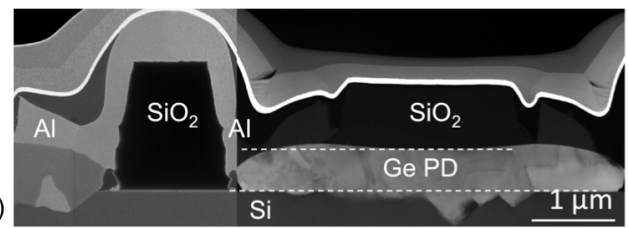

(b)

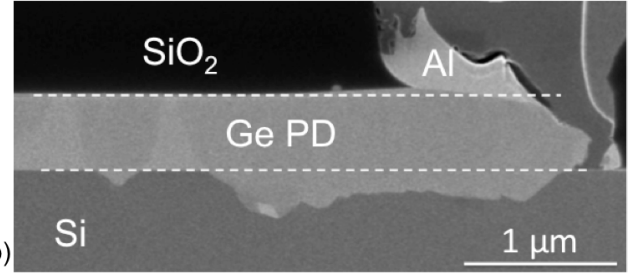

Fig. 3. (a) TEM image of a 16-diode hexapixel composed of $5 \times 5 \mu \mathrm{m}^{2}$ Ge-on-Si photodiodes. (b) TEM image of the edge of a single-diode pixel of $26 \times 26 \mu \mathrm{m}^{2}$.

windows was incomplete in regions near the $\mathrm{Al}$ tracks to the measurement pads. This was due to reflections from the Al during lithographical exposure of the resist. The $\mathrm{Al}$ remnants were much thinner that the as-deposited $\mathrm{Al}$, and the optical absorption at the long wavelengths used here was not of significance.

The most critical step in the processing was found to be the etching of the contact windows through the PECVD $\mathrm{SiO}_{2}$ with landing on the PureGaB layer [Fig. 1(c)]. The highresolution transmission electron microscopy (TEM) images, as shown in Fig. 3(a) and (b), revealed that the oxide is likely to be etched along the Ge-island perimeter down close to the Si substrate. This can result in the tip of Ge itselfwithout protection from the PureGaB layer-being exposed to the Al metallization. This phenomenon was studied in detail in [7]. A migration of $\mathrm{Al}$ through $\mathrm{Ge}$ was observed that induced a displacement of $\mathrm{Ge}$ and $\mathrm{Si}$, up to $5 \mu \mathrm{m}$ from the edge, resulting in $\mathrm{Ge}$-filled $\mathrm{V}$-grooves protruding into $\mathrm{Si}$ at the $\mathrm{Ge}-\mathrm{Si}$ interface. These grooves increased the vertical thickness of the Ge layer. As can be seen by comparing Fig. 3(a) and (b), it is clear that the Ge volume per diode area is larger for the multidiode devices both due to the larger thickness at the edge of the as-grown Ge crystal from loading effects and due to the Al-induced Ge migration. The thickness of $\mathrm{Ge}$ at the center of the $5 \times 5 \mu \mathrm{m}^{2}$ diode was $0.55 \mu \mathrm{m}$ while it was $<0.54 \mu \mathrm{m}$ for the $26 \times 26 \mu \mathrm{m}^{2}$ device. Therefore, while the surface area of the hexapixels was only $60 \%$ of that of the single-diode pixels, the volume per diode area increased due to the three times longer perimeter. This increase could be as high as $30 \%$.

\section{Electrical Characterization}

A continuous-flow cryostat system was used for achieving low temperatures. The cryogenic probe station (PMC150, SUSS MicroTech AG) was used for manually probing chips that were cooled in the cryostat system. Vacuum conditions were maintained to be free from ice and frosting on the chip surface even for temperatures as low as $77 \mathrm{~K}$ achieved with liquid nitrogen cooling. The Ge-on-Si photodiode samples were mounted with thermal grease onto a cold mount at the end of the refrigerator. This gives an excellent thermal contact between the sample and the fridge.
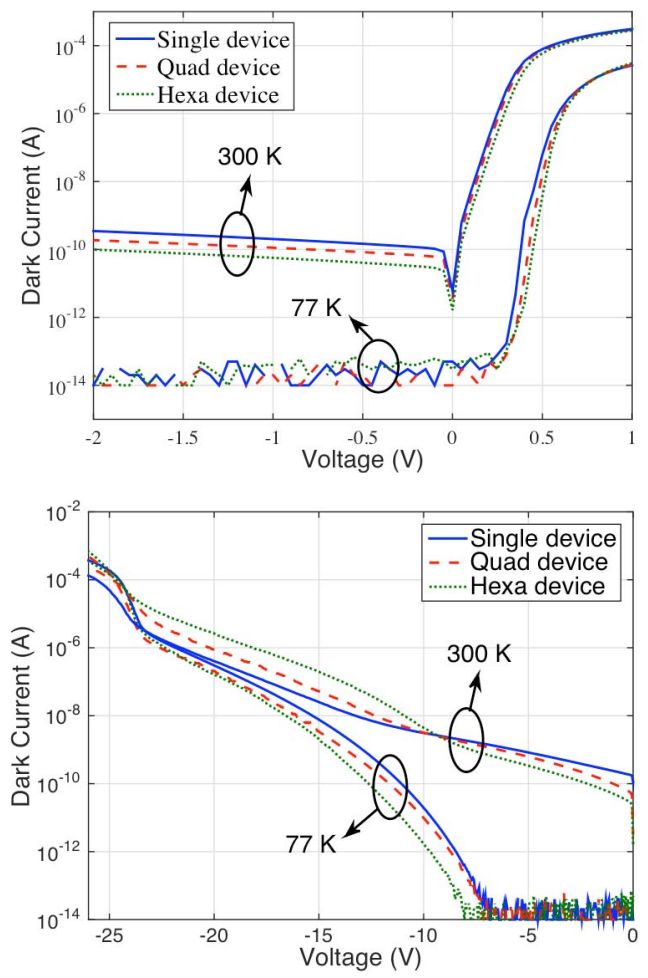

Fig. 4. $I-V$ characteristics at 300 and $77 \mathrm{~K}$ for the three pixel designs.

The diode $I-V$ characteristics were measured using a HP 4145B semiconductor parameter analyzer. Typical $I-V$ characteristics are shown in Fig. 4 for both room temperature and $77 \mathrm{~K}$ measurements of all three device types. The dark-current ranges from $1 \mathrm{nA}$ at room temperature to well under the measurement limit of the setup (10 fA) at $77 \mathrm{~K}$ at a reverse bias of up to $6 \mathrm{~V}$.

Ideally, the current would follow the Ge area as concluded in [7] on the basis of statistical measurements over the wafer. For devices with the lowest saturation current, compared with the single-diode device, the quad device had $\sim 30 \%$ lower current and the hexa device had $\sim 60 \%$ lower current. Over the arrays, a spread in currents that included values up to about a decade higher than the most ideal devices was identified, and could be related to the Al-migration through the $\mathrm{Ge}$ from the Al-exposed edge of the island.

The devices on the wafer that were chosen for the optical measurements discussed in this paper have a slightly higher n-doping of the Ge than those studied in [7], giving a slightly lower breakdown voltage of about $24 \mathrm{~V}$ instead of $30 \mathrm{~V}$. The spread in the $I-V$ characteristics is also more prominent over the whole wafer and less ideally low saturation currents were identified. This is particularly the case for the hexa devices that have a very large perimeter length. This can be seen from Fig. 5, where the $I-V$ characteristics at $77 \mathrm{~K}$ are shown for 70 devices from four different arrays of different pixel types. Nevertheless, while the hexapixels displayed relatively high current levels, the spread in current was lower than that of other pixel types. This can be understood in terms of the defects related to the parasitic processes at the $\mathrm{Ge}$-island perimeter. The perimeter of the hexa device was 3 times longer 

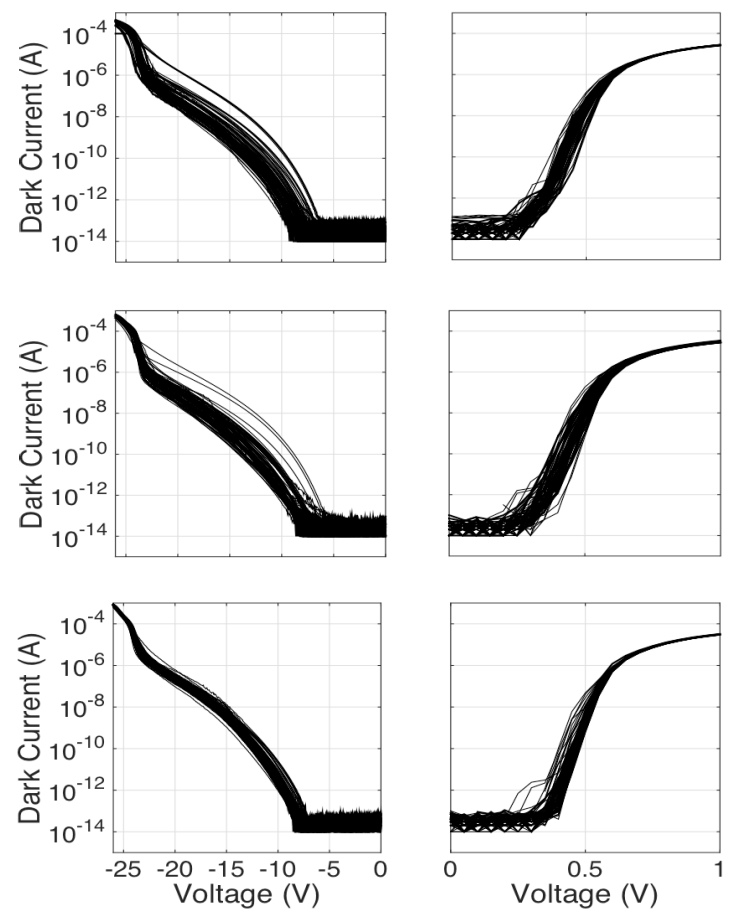

Fig. 5. Dark current (left) and forward current (right) spread across 70 devices measured at $77 \mathrm{~K}$. Top: single pixels. Middle: quad pixels. Bottom: hexapixels.

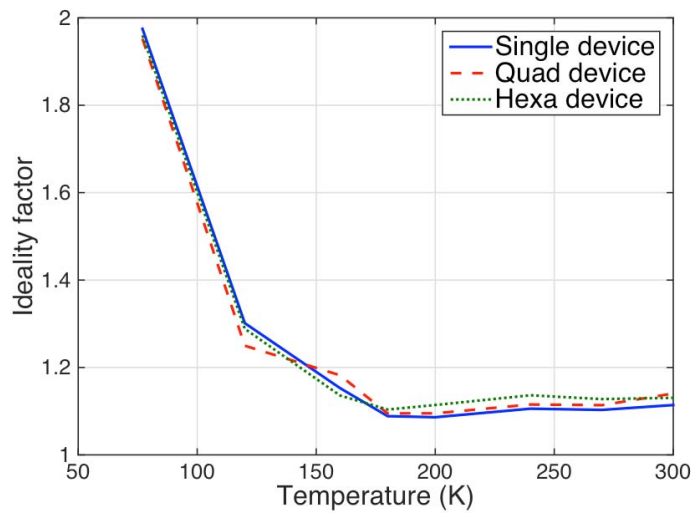

Fig. 6. Ideality factor for three different device types as a function of temperature.

than that of the single-diode pixel, i.e., it became less likely that a perfect device would be found.

The presence of defects that could become a source of nonideal currents was evident when considering the ideality factor $n$ given by

$$
I=I_{0}\left(e^{q V / n k T}-1\right)
$$

where $I_{0}$ is the saturation current and $q / k T$ is the thermal voltage at temperature $T$. With an increasing defect density, $n$ will increase from $n=1$ for ideal junctions to $n=2$ for junctions containing a high level of generation-recombination centers. In Fig. 6, $n$ is plotted as a function of temperature. The values are extracted from the slope of the forward $I-V$ characteristics, and were $\sim 1.12$ at room temperature for all three types of pixels despite the very long total perimeter of the hexa devices. Thus, it can be concluded that the electrically active defect density is low, even at the perimeter where Al-migration takes place. At $77 \mathrm{~K}$, the ideality factors increased to $\sim 2$, indicating that generation-recombination currents are dominating. Nevertheless, the low $n$ values at room temperature and very low dark currents substantiate that the Ge deposition, as well as the anode formation and contacting, are of good quality. The depletion width in reverse will quickly become larger than the $0.55 \mu \mathrm{m}$ thickness of the Ge-islands and transverse the nonperfect interface with the $\mathrm{Si}$. However, the distribution of dark current levels between the pixel types does not display any correlation to the decreasing Ge diode area when going from the single diode to hexa design, so that the Ge-to-Si interface defects do not appear to play any decisive role.

In all the cases, the device dark current was gradually increasing with reverse bias, leading to a gradual breakdown. Breakdown is governed by a large number of factors; in particular, the way the depletion region expands through $\mathrm{Ge}$ into the $\mathrm{Si}$ is of importance. The Ge thickness that varies from the edge of the island to the center will be decisive for the depletion width since the Ge doping is $\sim 10$ times higher than that of the Si substrate. Moreover, the Al-migration at the Ge-island edge will not only increase the Ge thickness but also result in coalescence of the Al into a small number of grains that can be found up to $\sim 5 \mu \mathrm{m}$ from the oxide edge. These can function as the Schottky diodes from which the n-region is also depleted [7]. When $\mathrm{Al}$ travels through $\mathrm{Ge}$ or $\mathrm{Si}$, it can also be incorporated as a p-dopant. In $\mathrm{Si}$, this was found to occur in the Al-induced solid-phase-epitaxy (SPE) process described in [13], by which the Si was doped to the Al solid solubility of $10^{18}-10^{19} \mathrm{~cm}^{-3}$ for anneal temperatures from $400{ }^{\circ} \mathrm{C}$ to $500{ }^{\circ} \mathrm{C}$. On $\mathrm{n}-\mathrm{Si}$, this $\mathrm{Al}$-doped SPE Si was used to form the anode of good quality $\mathrm{p}^{+} \mathrm{n}$ diodes [13]. The solid solubility of $\mathrm{Al}$ in $\mathrm{Ge}$ is much higher, about $4 \times 10^{20} \mathrm{~cm}^{-3}$ at $400{ }^{\circ} \mathrm{C}$ [14]. Therefore, not only $\mathrm{Al}$ grains but also Al-doped $\mathrm{p}^{+}$-regions can form junctions to the $\mathrm{n}-\mathrm{Ge}$ crystal. These phenomena could also account for the increase and spread in saturation current and reverse leakage current.

\section{OPTICAL CHARACTERIZATION}

The Ge photodiodes were mounted in the vacuum chamber and cooled to $77 \mathrm{~K}$. For accurate results, it is important that the photodiode arrays be uniformly exposed to light. Responsivity was measured at several wavelengths, using laser diodes as light sources (M660F1, M850F1, and M940F1 from THORLABS) with wavelengths of 660, 850, and $940 \mathrm{~nm}$, respectively. The corresponding output power of laser sources was $14.5,7.7$, and $6.5 \mathrm{~mW}$, respectively. However, the incident optical power on the devices was attenuated, resulting in 9.8, 0.75 , and $0.78 \mu \mathrm{W}$ for the respective laser sources. The diameter of the incident beam spot was $0.3 \mathrm{~cm}$.

The photocurrent was measured using a computer-controlled HP Semiconductor Parameter Analyzer model 4145B. A computer with ICCAP data acquisition was used to record the photocurrent through a general-purpose interface bus to the HP4145B. Neutral density filters were used to prevent light saturation and pileup. 


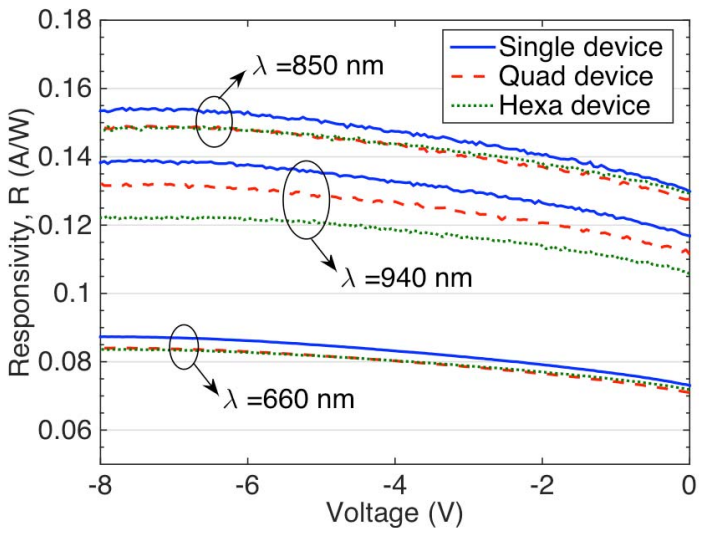

Fig. 7. Responsivity $R$ measured at $77 \mathrm{~K}$ for three different device types.

TABLE II

Summary of Overall Best Performance Compared With Several Ge Photodiode Designs in the Literature

\begin{tabular}{|c|c|c|c|c|c|}
\hline & $\begin{array}{l}\text { PureGaB } \\
\text { Ge diode }\end{array}$ & $\begin{array}{l}\text { Ref } \\
{[17]}\end{array}$ & Ref [18] & $\operatorname{Ref}[19]$ & $\begin{array}{l}\text { Ref } \\
{[20]}\end{array}$ \\
\hline $\begin{array}{c}\text { Ge Thickness } \\
(\mu \mathrm{m})\end{array}$ & 0.6 & 1.1 & 0.4 & Bulk Ge & 2 \\
\hline $\begin{array}{c}\text { Dark current } \\
\text { density } \\
\left(\mu \mathrm{A} / \mathrm{cm}^{2}\right) \\
@-1 \mathrm{~V}, 300 \mathrm{~K}\end{array}$ & 35 & 19 & 1500 & 62 & $\begin{array}{l}400 @ \\
-2 \mathrm{~V}, \\
263 \mathrm{~K}\end{array}$ \\
\hline $\begin{array}{c}\mathrm{R}(\mathrm{A} / \mathrm{W}) \\
@ 77 \mathrm{~K}\end{array}$ & $\begin{array}{c}0.150 \\
@ 850 \mathrm{~nm} \\
0.135 \\
@ 940 \mathrm{~nm} \\
\end{array}$ & $\begin{array}{c}0.55 \\
@ 1300 \\
\mathrm{~nm}\end{array}$ & $\begin{array}{c}0.18 \\
@ 850 \mathrm{~nm}\end{array}$ & $\begin{array}{c}0.55 \\
@ 1550 \\
\mathrm{~nm}\end{array}$ & - \\
\hline $\begin{array}{l}\text { QE (\%) } \\
\text { @77 K }\end{array}$ & $\begin{array}{c}21.9 \% \\
@ 850 \mathrm{~nm} \\
17.8 \% \\
@ 940 \mathrm{~nm}\end{array}$ & $\begin{array}{c}52 \% \\
@ 1300 \\
\mathrm{~nm}\end{array}$ & $\begin{array}{c}27 \% \\
@ 850 \mathrm{~nm}\end{array}$ & $\begin{array}{c}45 \% \\
@ 1550 \\
\mathrm{~nm}\end{array}$ & $\begin{array}{c}42 \% \\
@ 1300 \\
\mathrm{~nm}, \\
193 \mathrm{~K}\end{array}$ \\
\hline
\end{tabular}

The responsivity $R$ as a function of reverse voltage $V_{x}$ was calculated as

$$
R\left(V_{x}\right)=\left(I_{\mathrm{ph}, V_{x}}-I_{\mathrm{dark}, V_{x}}\right) / P
$$

where the photocurrent $I_{\mathrm{ph}, V_{x}}$ and the dark current $I_{\mathrm{dark}, V_{x}}$ are measured at $V_{x}$ and $P$ is the incident power at the surface of the pixel. In Fig. 7, the extracted $R$ at $77 \mathrm{~K}$ is plotted for all three types of devices and available wavelengths. The $P$ is calculated assuming a pixel area of $26 \times 26 \mu \mathrm{m}^{2}$ in all cases. The responsivity values are comparable with what is commonly found for Ge detectors, a few values for which are listed in Table II and compared with our single diode $26 \times 26 \mu \mathrm{m}^{2}$ device. The responsivity generally increases with wavelength, and the peak responsivity in Ge is found at a wavelength slightly lower than the cutoff wavelength at $\sim 1.6 \mu \mathrm{m}$. Nevertheless, here, $R$ at $940 \mathrm{~nm}$ is slightly lower than at $850 \mathrm{~nm}$. This behavior can be explained in terms of the absorption length of light in Ge that is 100,320 , and $500 \mathrm{~nm}$ for the incident wavelengths of 660,850 , and $940 \mathrm{~nm}$, respectively. The absorption increases with increasing depletion of the $\mathrm{Ge}$, as can be seen here for a voltage range from 0 to $\sim-6 \mathrm{~V}$. At about $-6 \mathrm{~V}$, the $\mathrm{Ge}$ is completely depleted. The absorption in $\mathrm{Si}$ is negligible compared with that

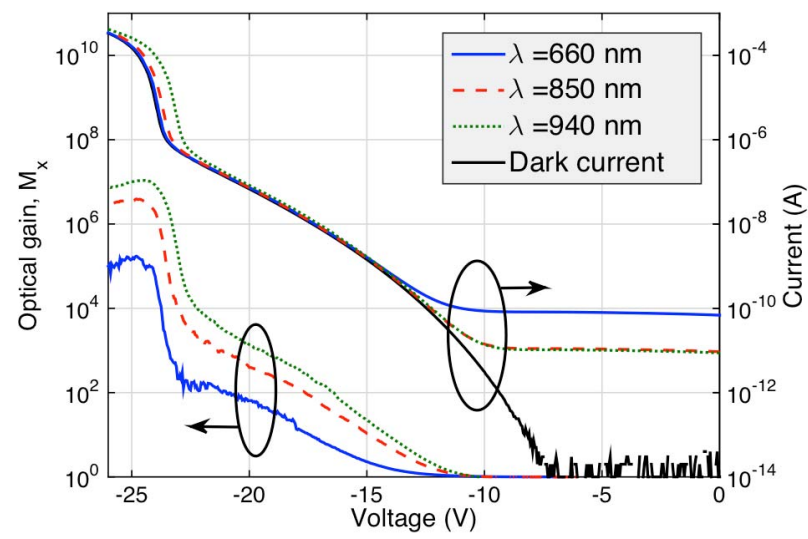

Fig. 8. Optical gain as a function of reverse voltage for three different wavelengths at $77 \mathrm{~K}$ for a single-diode pixel, and the corresponding $I-V$ characteristics for both the illuminated and nonilluminated cases.

in Ge, hence with the 500-nm absorption length of 940-nm light, a relatively large amount of light will pass through the Ge without being detected. At $0 \mathrm{~V}, 660$ - and 850 -nm light resulted in photocurrents with essentially no device geometry dependence, and the quad and hexa devices maintain the same photocurrent through to $-8 \mathrm{~V}$. At this voltage, however, the single-diode device had a nearly $5 \%$ higher photocurrent. For a wavelength of $940 \mathrm{~nm}$, the increase was $\sim 10 \%$. Considering that the Ge-diode area is almost halved when going from the single diode to the hexa device, the small variation of the photocurrent with area suggests that a significant amount of stray peripheral light is picked up at the diode perimeter, separated by only $1-\mu \mathrm{m}$-wide oxide hills in the multidiode devices. Another factor that may play a role is the higher Ge thickness at the perimeter of the diodes but if this was the decisive factor a larger increase in $R$ would be expected with increasing $V_{x}$ that almost doubles the Ge volume.

The gradual breakdown of the diodes makes them suitable for operation as APDs. The high sensitivity of an APD relies on using avalanche multiplication to generate an internal signal gain. In this mode, the absorbed photon generates electron-hole pairs that are multiplied thus increasing the initial photocurrent flow. The total current was measured for the three types of Ge-on-Si photodiodes with the reverse bias voltage going from 0 to $-26 \mathrm{~V}$ while the devices were illuminated by the laser.

The optical gain $M_{x}$ at reverse voltage $V_{x}$ was calculated here as

$$
M_{x}=\left(I_{\mathrm{ph}, V_{x}}-I_{\mathrm{dark}, V_{x}}\right) /\left(I_{\mathrm{ph}, 0}-I_{\mathrm{dark}, 0}\right)
$$

where $I_{\mathrm{ph}, V_{x}}$, and $I_{\mathrm{dark}, V_{x}}$ were measured at a high reverse voltage $V_{x}$ while $I_{\mathrm{ph}, 0}$ and $I_{\mathrm{dark}, 0}$ were measured at a small reverse bias of $-7 \mathrm{~V}$, where the depletion over the junction covers the whole Ge crystal but the leakage current is not yet influenced by impact ionization. The photocurrent for different illuminations and the dark current, both measured at $77 \mathrm{~K}$ for the $26 \times 26 \mu \mathrm{m}^{2}$ single-diode device, are shown in Fig. 8, along with $M_{x}$ that peaks at $-24 \mathrm{~V}$ with more than $10^{6}$ at wavelengths of 940 and $850 \mathrm{~nm}$. This underlines the fact that the dark current, even when impact ionization events 

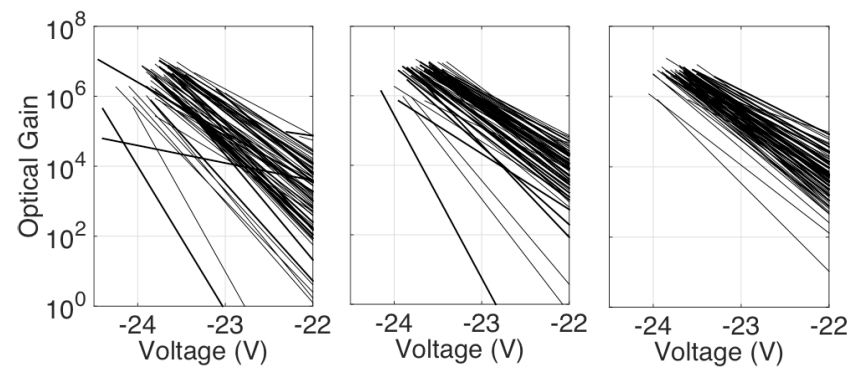

Fig. 9. Fitted optical gain spread across 70 devices measured at $940 \mathrm{~nm}$ and $77 \mathrm{~K}$. Left: single pixels. Center: quad pixels. Right: hexapixels.

dominate, is very low. In line with a higher responsivity at the NIR wavelengths, the gain also displayed higher values than at visible wavelengths, with $940 \mathrm{~nm}$ yielding the highest gain. This suggests that the photoevents occurring near the metallurgic junction, where the electric field is highest, create more electron-hole pairs than deeper photoevents, presumably because not only the holes, but also the electrons, will achieve a high velocity when created in the high field region. The responsivity was measured at low voltage where it was slightly lower at $940 \mathrm{~nm}$ than at $850 \mathrm{~nm}$ because of the limited thickness of the $\mathrm{Ge}$ islands. In bulk $\mathrm{Ge}$, however, the responsivity generally will increase linearly with wavelength at least up to $1000 \mathrm{~nm}$ so if multiplication is highest at the surface, the $940 \mathrm{~nm}$ wavelength will give the highest gain. This effect is an advantage for the targeted applications in the NIR region and may improve performance at wavelengths up to $1.6 \mu \mathrm{m}$ despite the limited Ge thickness.

The responsivity at $660 \mathrm{~nm}$ is mainly not reported for $\mathrm{Ge}$ photodiodes. In other types of devices with tens-ofnanometer-thick implanted or epitaxially grown light-entrance windows, it will also be very low because of the low absorption length in $\mathrm{Ge}$ at visible wavelengths [15]. For our PureGaB devices where the $\mathrm{p}^{+}$-anode is only a few nanometer thick, the optical gain and responsivity remains high even at $660 \mathrm{~nm}$, about $0.085 \mathrm{~A} / \mathrm{W}$, which is about the same as the responsivity of the PureB Si photodiodes at a wavelength of $400 \mathrm{~nm}$ where the penetration depth also reduces to $100 \mathrm{~nm}$ [16].

The leakage current of the devices is related to the defect density that may also influence the impact ionization process. To evaluate whether there was a connection, the gain-to- $V_{\mathrm{bd}}$ relationship was determined for 70 devices of each geometry from Fig. 5. Lin-log fits of the optical gain as a function of voltages close to breakdown are shown in Fig. 9. They reveal a spread that appears to be smallest for the hexa devices; nevertheless, the gain is comparable for all geometries. The difference in optical gain from device type to device type is, therefore, likely to be related to the spread in dark current rather than to the geometrical effects.

This trend is corroborated by Fig. 10, which plots the maximum optical gain as a function of $V_{\mathrm{bd}}$. From this plot, it is clear that, although the breakdown voltage is similar for all three pixel geometries, the spreading is lowest for the hexapixel array. This is also true for the spread in maximum optical gain. The standard deviations are represented in Fig. 10 by the
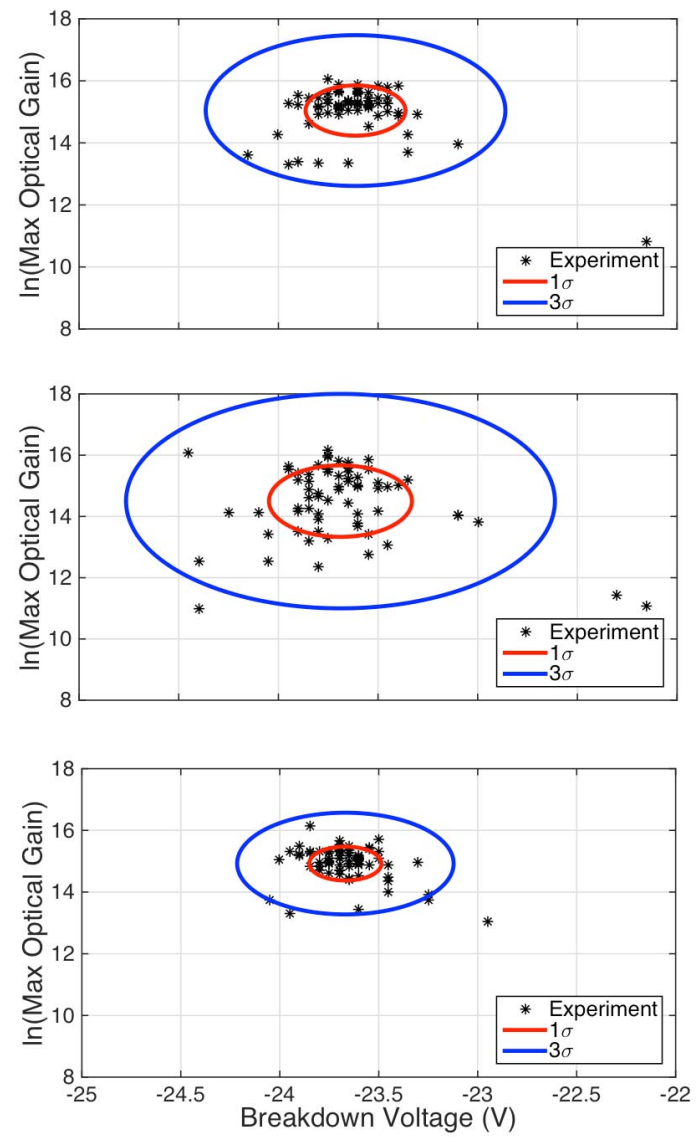

Fig. 10. Spreading of maximum optical gain versus breakdown voltage across 70 devices measured at $940 \mathrm{~nm}$ and $77 \mathrm{~K}$. Top: single pixels. Middle: quad pixels. Bottom: hexapixels.

TABLE III

SUMMARY OF APD PERFORMANCE PARAMETERS FOR THE THREE PIXEL TYPES

\begin{tabular}{|c|c|c|c|}
\hline & $\begin{array}{c}\text { Mean value of } \\
V_{b d}\end{array}$ & $\sigma\left(V_{b d}\right)$ & $\begin{array}{c}\text { Mean of max. } \\
\text { optical gain }\end{array}$ \\
\hline Single & $-23.69 \mathrm{~V}$ & $0.36 \mathrm{~V}$ & $3.18 \times 10^{6}$ \\
\hline Quad & $-23.61 \mathrm{~V}$ & $0.25 \mathrm{~V}$ & $4.16 \times 10^{6}$ \\
\hline Hexa & $-23.67 \mathrm{~V}$ & $0.18 \mathrm{~V}$ & $3.42 \times 10^{6}$ \\
\hline
\end{tabular}

inner ellipse for $\sigma$ and the outer ellipse for $3 \sigma$ spread. The mean values of maximum optical gain and breakdown, as well as the breakdown standard deviation, are listed in Table III. The spreading of $V_{\mathrm{bd}}$ was $1.52 \%, 1.07 \%$, and $0.78 \%$ for single pixels, quadpixels, and hexapixels, respectively. Just as the lower spread around high current levels of the reverse $I-V$ characteristics of the hexa devices can be explained by the higher probability of perimeter imperfections, this also explains the lower spread with perimeter of the maximum gain to breakdown voltage relationship.

\section{CONClusion}

The PureGaB Ge-on-Si photodiodes integrated in $300 \times 1$ pixel arrays were characterized at cryogenic temperatures for operation as proportional APDs. Three different pixel geometries were investigated. The main 
differentiating factor is a very different area and perimeter, while the electrical/optical performance is comparable with very high optical gain of up to $10^{6}$ measured at $77 \mathrm{~K}$, where the low-voltage dark current is lower than the measurement limit of $2.5 \times 10^{-2} \mu \mathrm{A} / \mathrm{cm}^{2}$. When measured at $77 \mathrm{~K}$, the breakdown voltage spread is low $(23.6 \pm 0.4 \mathrm{~V})$, and the quantum efficiency reaches up to $21.9 \%$ at the wavelength of $850 \mathrm{~nm}$. The values of responsivity in all three designs decreased by $5 \%$ when going from the 850 to $940 \mathrm{~nm}$ wavelength due to the decrease in absorption coefficient combined with the limited Ge thickness of $\sim 0.6 \mu \mathrm{m}$.

The hexa design, composed of the smallest individual Ge-on-Si diodes, had the smallest total $\mathrm{Ge}$ area but the largest perimeter with an average increase in the Ge thickness of $\sim 30 \%$ due mainly to V-groove formation. This effect increases the dark current levels but appears to provide the most desirable performance at cryogenic temperatures in terms of spread in the dark current and the optical gain.

The limited Ge thickness enables a very flat topography that is beneficial for integration in the CMOS. All processing steps are performed with high reliability on equipment already in use in the CMOS production but TMGa is not normally installed on $\mathrm{Si} / \mathrm{SiGe}$ epitaxy equipment. However, only a small modification of the system is needed for adding Ga deposition to the equipment capabilities. All in all, the PureGaB Ge-on-Si offers a very low-complexity CMOS-compatible means of fabricating uniform arrays of the NIR sensitive photodetectors that are operational in linear, avalanche, and even Geiger modes.

\section{ACKNOWLEDGMENT}

The authors would like to thank W. Grabinski and Prof. A. Ionescu from the Nanoelectronic Devices Group at École Polytechnique Fédérale de Lausanne, Lausanne, Switzerland, and ID Quantique SA for electrical and optical measurement support, and the staff of the DIMES cleanrooms for processing support.

\section{REFERENCES}

[1] M. Aminian, A. Sammak, L. Qi, L. Nanver, and E. Charbon, "A Ge-on-Si single-photon avalanche diode operating in Geiger mode at infrared wavelengths," Proc. SPIE, vol. 8375, p. 83750Q, May 2012.

[2] V. Saveliev, "The recent development and study of silicon photomultiplier," Nucl. Instrum. Methods Phys. Res. A, Accel. Spectrom., Detectors Assoc. Equip., vol. 535, nos. 1-2, pp. 528-532, Dec. 2004.

[3] E. Charbon, "Single-photon imaging in complementary metal-oxidesemiconductor processes," Philos. Trans. Roy. Soc. London A, Math Phys. Eng. Sci., vol. 372, no. 2012, p. 20130100, 2014.

[4] A. Tosi, A. D. Mora, S. Tisa, F. Acerbi, F. Zappa, and S. Cova, "InGaAs/InP SPADs for near-infrared applications: Device operating conditions and dedicated electronics," Proc. SPIE, vol. 7681, p. 76810R, Apr. 2010.

[5] L. Vivien et al., " $42 \mathrm{GHz}$ p.i.n Germanium photodetector integrated in a silicon-on-insulator waveguide," Opt. Exp., vol. 17, no. 8, pp. $6252-6257,2009$

[6] G. Masini, V. Cencelli, L. Colace, F. De Notaristefani, and G. Assanto, "Monolithic integration of near-infrared Ge photodetectors with $\mathrm{Si}$ complementary metal-oxide-semiconductor readout electronics," Appl. Phys. Lett., vol. 80, no. 18, p. 3268, 2002.

[7] A. Sammak, L. Qi, and L. K. Nanver, "Restricted-access Al-mediated material transport in $\mathrm{Al}$ contacting of PureGaB Ge-on-Si p+n diodes," J. Electron. Mater, to be published.

[8] A. Sammak, M. Aminian, L. Qi, W. B. De Boer, E. Charbon, and L. K. Nanver, "Fabrication of PureGaB Ge-on-Si photodiodes for well-controlled 100-pA-level dark currents," Electro-Chem. Soc. Trans., vol. 64, no. 6, pp. 737-745, 2014.
[9] A. Sammak, M. Aminian, L. Qi, E. Charbon, and L. K. Nanver, "A $270 \times 1$ Ge-on-Si photodetector array for sensitive infrared imaging," Proc. SPIE, vol. 9141, p. 914104, May 2014.

[10] A. Sammak, M. Aminian, L. Qi, W. B. De Boer, E. Charbon, and L. K. Nanver, "A CMOS compatible Ge-on-Si APD operating in proportional and Geiger modes at infrared wavelengths," in Proc. IEDM, Washington, DC, USA, Dec. 2011, pp. 8.5.1-8.5.4.

[11] I. P. Santos, P. J. Caspers, T. B. Schut, R. V. Doorn, S. Koljenović, and G. J. Puppels, "Implementation of a novel low-noise InGaAs detector enabling rapid near-infrared multichannel Raman spectroscopy of pigmented biological samples," J. Raman Spectrosc., to appear, 2015.

[12] A. Sammak, W. B. De Boer, and L. K. Nanver, "Ge-on-Si: Single-crystal selective epitaxial growth in a CVD reactor," Electro-Chem. Soc. Trans., vol. 50, no. 9, pp. 507-512, 2012.

[13] Y. Civale, L. K. Nanver, P. Hadley, E. J. G. Goudena, and H. Schellevis, "Sub-500 ${ }^{\circ} \mathrm{C}$ solid-phase epitaxy of ultra-abrupt $\mathrm{p}^{+}$-silicon elevated contacts and diodes," IEEE Electron Device Lett., vol. 27, no. 5, pp. 341-343, May 2006.

[14] C. Belove, Ed., Handbook of Modern Electronics and Electrical Engineering. New York, NY, USA: Wiley, 1986.

[15] R. E. Warburton et al., "Ge-on-Si single-photon avalanche diode detectors: Design, modeling, fabrication, and characterization at wavelengths 1310 and 1550 nm," IEEE Trans. Electron Devices, vol. 60, no. 11 , pp. 3807-3813, Nov. 2013.

[16] L. K. Nanver et al., "Robust UV/VUV/EUV pureB photodiode detector technology with high CMOS compatibility," IEEE J. Sel. Topics Quantum Electron., vol. 20, no. 6, Nov./Dec. 2014, Art. ID 3801711.

[17] Y. Kang et al., "Monolithic germanium/silicon avalanche photodiodes with $340 \mathrm{GHz}$ gain-bandwidth product," Nature Photon., vol. 3, no. 1, pp. 59-63, 2009.

[18] T. H. Loh et al., "Selective epitaxial germanium on silicon-on-insulator high speed photodetectors using low-temperature ultrathin $\mathrm{Si}_{0.8} \mathrm{Ge}_{0.2}$ buffer," Appl. Phys. Lett., vol. 91, no. 7, p. 073503, 2007.

[19] M. Takenaka, K. Morii, M. Sugiyama, Y. Nakano, and S. Takagi, "Dark current reduction of $\mathrm{Ge}$ photodetector by $\mathrm{GeO}_{2}$ surface passivation and gas-phase doping," Opt. Exp., vol. 20, no. 8, pp. 8718-8725, 2012.

[20] I. Åberg et al., "A low dark current and high quantum efficiency monolithic germanium-on-silicon CMOS imager technology for day and night imaging applications," in Proc. IEDM, San Fransisco, CA, USA, Dec. 2010, pp. 14.4.1-14.4.4

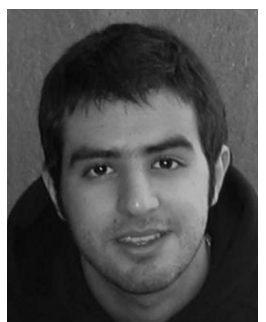

Amir Sammak was born in Tehran, Iran, in 1983. He received the B.Sc. and M.Sc. degrees in electrical engineering from the University of Tehran, Tehran, in 2005 and 2007, respectively, and the Ph.D. degree from the Faculty of Electrical Engineering, Mathematics and Computer Science, Delft University of Technology, Delft, The Netherlands, in 2012.

$\mathrm{He}$ is currently a Post-Doctoral Research Fellow with the Delft University of Technology.

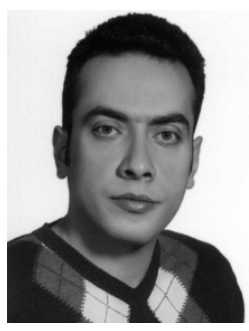

Mahdi Aminian was born in Tehran, Iran $\mathrm{He}$ received the bachelor's degree in computer engineering and the master's degree in computer architecture from the Amirkabir University, Tehran, in 2006 and 2008, respectively, and the $\mathrm{Ph} . \mathrm{D}$. degree from the Department of Electrical Engineering, École Polytechnique Fédérale de Lausanne, Lausanne, Switzerland, in 2014.

$\mathrm{He}$ is currently involved in Ge-on-Si single photon image sensors and Si deep junction sensors for near-infrared detection. 


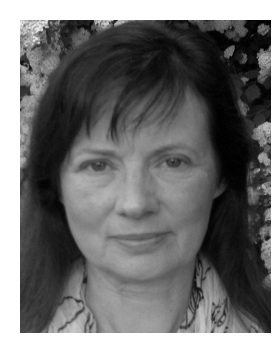

Lis K. Nanver (M'80) received the master's degree in physics from the University of Aarhus, Aarhus, Denmark, in 1979, and the Doctor-Engineer degree in physics applied to telecommunications from the Ecole Nationale Supérieure des Télécommunications, Paris, France, in 1982, and the Ph.D. degree in electrical engineering from the Delft University of Technology, Delft, The Netherlands, in 1987.

She is currently with Aalborg University, Aalborg, Denmark and also the University of Twente, Enschede, The Netherlands.

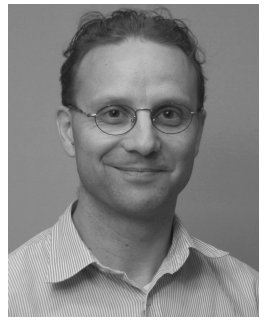

Edoardo Charbon (M'92-SM'10) received the Diploma degree from ETH Zurich, Zurich, Switzerland, in 1988, the M.S. degree from the University of California at San Diego, La Jolla, in 1991, and the Ph.D. degree from the University of California at Berkeley, Berkeley, CA, USA, in 1995, all in electrical engineering and electrical engineering and computer sciences.

$\mathrm{He}$ has been with the École Polytechnique Fédérale de Lausanne, Lausanne, Switzerland, since 2002 and Delft University of Technology, Delft, 